\title{
Thermal rate constants of beryllium clusters in gas phase
}

\author{
JánMatúška ${ }^{a}$, Ivan Sukuba ${ }^{b}$ \\ ${ }^{a}$ Institute of Physical Chemistry and Chemical Physics, FCHPT STU, Radlinského 9, Bratislava, Slovakia \\ ${ }^{b}$ Department of Nuclear Physics and Biophysics, Faculty of Mathematics, Physics and Informatics, \\ Comenius University, SK-84248, Bratislava, Slovakia \\ jan.matuska@stuba.sk
}

\begin{abstract}
Formation and fragmentation of beryllium clusters up to 100 atoms were studied using molecular dynamics trajectory simulations. Association rate constants of a single $\mathrm{Be}$ atom attachment to $\mathrm{Be}_{n}$ clusters, $n=2-20,30,40,50,60,70,80,90$ and 100, and dissociation rate constants of these clusters were calculated directly. Dependence of the dissociation rate constants on temperature is strong and follows the Arrhenius equation. An analysis of the dissociation reactions has shown that beryllium clusters dissociate mainly into single Be atoms. The monomer association reaction depends weakly on temperature but strongly on the size of the cluster.
\end{abstract}

Keywords: beryllium clusters, molecular dynamics, thermal equilibrium rate constants

\section{Introduction}

The main goal of the international project ITER (Pitts et al., 2011) is to develop and build a demonstration fusion energy power plant fueled by the fusion reaction between hydrogen isotopes, deuterium and tritium. This fusion occurs in hot plasma with the temperature of 150 million $\mathrm{K}$ confined by a strong magnetic field within the reactor based on the tokamak concept, a toroidal shaped device. Unfortunately, inner walls are never fully protected from the plasma. The flow of plasma particles, neutrons, and heat through the magnetic field caused by the turbulence of the edge plasma damages the blanket. Special, detachable first walls are planned for the protection of the blanket facing the incoming particles and heat thus minimizing unwanted damage. Beryllium is planned to be used for the blanket in a vacuum vessel of the tokamak device as the protection against fusion-plasma due to its unique chemical and physical properties; it is often referred to as a plasma-facing component (PFC).

At conditions that beryllium parts have to withstand during the runtime, like heat loads or high particle fluxes, diverse processes and interactions take place resulting in erosion of these materials. Initial starters are the impinging particles from plasma and the waste accumulated in the divertor. The further processes range from sputtering and erosion of material, retention, to forming of new alloys with various undesired properties and dust formation. Furthermore, too fixed, expensive, and regulated experiments due to beryllium toxicity are amongst the disadvantages of experimental research and result in increased use of computational material science and theoretical approaches for the simulation and modeling of these processes to explain physics behind them. Still, significant progress in experimental as well as theoretical research on beryllium concerning fusion development has been made. Research done in the Joint European Torus (JET) tokomak (Matthews et al., 2011; Brezinsek, 2011; Litaudon, 2017) and the PISCES-B facility (Doemer et al., 2009; Nishijima et al., 2009) contributed substantially by experiments with beryllium to the knowledge of the important processes like sputtering and $\mathrm{D}_{2}$ outgassing which serves further as input data for transport codes like ERO (Widdowson et al., 2014), together with other theoretically obtained data, like rate constants, electron-impact cross sections of small beryllium clusters and sputtering yields or surface binding energy of Be surfaces, using ab initio quantumchemical methods and molecular dynamics simulations (Sun and Fournier, 2005; Safi et al., 2017; Gyoeroek et al., 2016) to uncover the underlying principles of these processes.

Concerning this topic and the use of MD for small metal clusters, $\mathrm{Li}$ and Truhlar ( $\mathrm{Li}$ and Truhlar, 2008) studied the association reactions of the $\mathrm{Al}$ atom with $\mathrm{Al}_{n}$ particles and the unimolecular dissociation of $\mathrm{Al}_{n}$ by classical molecular dynamics trajectory simulations and determined thermal reaction rate constants for both reaction types.

In the present study, molecular dynamics simulations to determine the association (forward) and dissociation (backward) thermal rate constants for beryllium clusters $\mathrm{Be}_{n}, n=2-20,30,40,50,60,70$, 80, 90, 100 were performed.

\section{Theory and computational details}

Processes of formation (association) and fragmentation of beryllium clusters have been studied 
employing the reaction schemes:

$$
\begin{gathered}
\mathrm{Be}+\mathrm{Be}_{n-1} \rightleftharpoons \mathrm{Be}_{n} \text { association } \\
\mathrm{Be}_{n} \rightleftharpoons \mathrm{Be}_{m}+\mathrm{Be}_{n-m} \text { fragmentation }
\end{gathered}
$$

where $\mathrm{Be}_{n}$ clusters are formed by 2-20, 30, 40, 50, $60,70,80,90$ and 100 beryllium atoms. In general, the association and dissociation rate constants depend on the concentration of the third body particles (Blais and Truhlar, 1983). The third body was substituted by a thermostat enabling the addition or removal of the energy from the system necessary to run the reaction. Molecular dynamics simulations were carried out applying the ANT 16 computational code (Zheng, 2016) using the Liouville formulation of the velocity Verlet integrator (Tuckerman et al., 1992) with a time step of 2.0 fs. All simulations were performed at a constant temperature controlled by a two-chain Nosé-Hoover thermostat (Martyna et al., 1992). Vibrational frequency of the thermostat was $0.05 \mathrm{fs}^{-1}$. Interatomic interactions were described by the analytical bond-order potential with a set of parameters for Be I (Björkas et al., 2009), i.e. the cut-off for interactions, $R_{\text {cut }}$, is $2.908 \AA$. Structures of local minima used for the trajectory calculations were obtained from randomly optimized structures by simulated annealing in the range from $10000 \mathrm{~K}$ to $1000 \mathrm{~K}$ and subsequently optimized using the BFGS algorithm (Byrdet et al., 1995) at randomly selected intermediate steps. The six lowest energy structures were considered as the local minima and used in further simulations. The dependence of the cohesive energy on the size of the cluster (see Fig. 1) shows a convergence $\left(\sim-2.75 \mathrm{eV}\right.$ for $\left.\mathrm{Be}_{100}\right)$. The value for bulk beryllium varies with the structure. Our structures are packed closer than simple cubic ones $(-2.31 \mathrm{eV})$ but they do not reach the values of the body centered cubic, face centered cubic or hexagonal close-packed $(-3.27 \mathrm{eV},-3.16 \mathrm{eV}$, -3.32 eV, respectively) structures (Bjorkas et al., 2009). These structures were used to generate the initial conditions of the simulation considering classical phase space distribution of separable harmonic oscillators and they were equilibrated for $20 \mathrm{ps}$ at the target temperature: from $1500 \mathrm{~K}$ to $2750 \mathrm{~K}$ for $\mathrm{Be}_{1}-\mathrm{Be}_{10}$, from $1750 \mathrm{~K}$ to $2750 \mathrm{~K}$ for $\mathrm{Be}_{11}-\mathrm{Be}_{20}$ and from $2250 \mathrm{~K}$ to $2750 \mathrm{~K}$ for the rest of the clusters. At temperatures below $1500 \mathrm{~K}$, no significant reactions within the studied time frame were observed. After the equilibration, a total of $N_{\text {traj }}, N_{\text {traj }}=10000$, sets of coordinates and momenta were randomly selected and saved as the initial configurations for the simulation of association and dissociation reactions. The starting configurations obtained by this procedure are not completely uncorrelated because they are calculated from the same initial minimum-energy structure and they are close to each other in the phase space. The procedure was repeated for each local minimum to obtain uncorrelated results. The reported rate constants are represented by the average value of six rate constants obtained from six different sets of trajectory simulations.

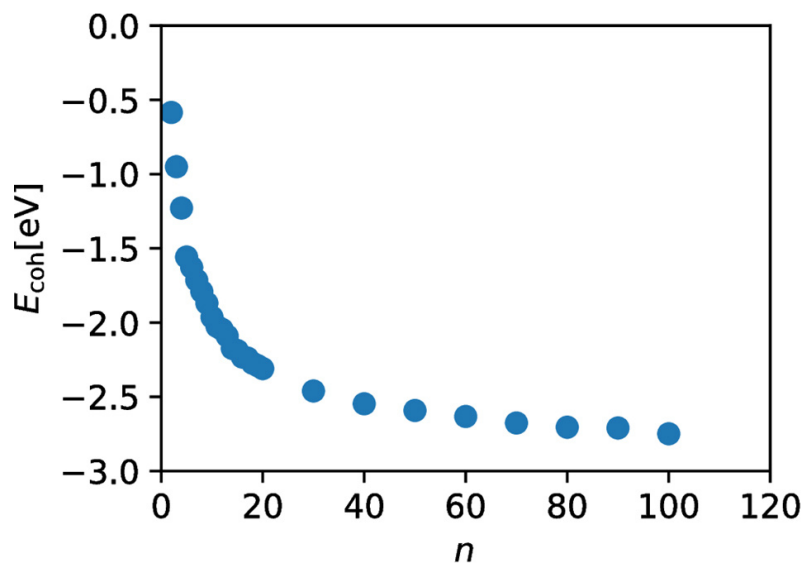

Fig. 1. Dependence of cohesion energy, $E_{\text {coh }}$, on the size of the cluster, $n$.

Rate constant of the monomer association reaction (Eq. 1) was obtained from a trajectory calculation. For each of the initial conditions of the rotating $\mathrm{Be}_{n-1}$ cluster (target), collisions with a Be atom were simulated. The thermal rate constant was obtained by Monte Carlo sampling over all initial conditions including the relative translational energy and the impact parameter between two reactants. Then, the association rate constant, $k_{1, n}^{\text {Ass }}$, is given as:

$$
k_{1, n}^{\mathrm{Ass}}=\sqrt{\frac{8 k_{B} T}{\mu}} b_{\mathrm{max}}^{2} P_{r(1, n)}^{\mathrm{Ass}}
$$

Where $k_{\mathrm{B}}$ is the Boltzmann constant, $\mu$ reduced mass, $b_{\max }$ is the maximum impact parameter, and $P_{r(1, n)}^{\text {Ass }}$ the fraction of reactive trajectories:

$$
P_{r(1, n)}^{\text {Ass }}=\frac{N_{r(1, n)}^{\text {Ass }}}{N_{\text {traj }}}
$$

where $N_{r(1, n)}^{\text {Ass }}$ is the number of reactive trajectories. The value of $b_{\max }$ is determined in the following way:

$$
b_{\max }=2.2\left(d_{\text {max }} / 2+R_{\text {cut }}\right)
$$

where $d_{\max }$ is the maximum distance between the atoms in the target particle and $R_{\text {cut }}$ is the maximal potential cutoff radius.

The reaction is considered reactive if two conditions are fulfilled. The first one defines, according to Stillinger, that the particle belongs to the cluster if the interatomic distance between the particle 
and at least one of atoms of the cluster is less than $R_{\text {cut }}$ (Stillinger, 1963). The second one states that the projection of the relative velocity between the two reactants on the vector of their center-of-mass separation changes the sign more than three times. At very high temperatures, the cluster may dissociate into fragments before the atom collides with it, in which case, the trajectory is not counted as associative, i.e. not counted in $N_{r(1, n)}^{\text {Assoc }}$ and $N_{\text {traj }}$, and is excluded.

Rate constant of the fragmentation reaction (Eq. 2) was calculated by monitoring the time evolution of the rotating $\mathrm{Be}_{n}$ cluster for each of the initial conditions. The cluster is considered dissociated when the Stillinger's criterion is not met in 50 successive time steps. Then, the time dependence of the dissociation probability is defined:

$$
P_{r}^{\text {Frag }}(t)=\frac{N_{r}^{\text {Frag }}(t)}{N_{\text {traj }}}
$$

where $N_{r}^{\text {Frag }}(t)$ is the number of dissociative trajectories in time $t$. In general, the dependence of the dissociation probability on time is exponential and can be described by:

$$
\ln \left(1-P_{r}^{\text {Frag }}(t)\right)=-t k_{n}^{\text {Frag }}
$$

where $k_{n}^{\text {Frag }}$ is the total dissociation rate constant which can be obtained by linear-least-square fitting of equation (7). The rate constant of an individual channel can be obtained as:

$$
k_{m, n}^{\text {Frag }}=f_{m, n}^{\text {Frag }} k_{n}^{\text {Frag }}
$$

where $f_{m, n}^{\text {Frag }}$ is the fragmentation-channel probability, the ratio of the number of trajectories dissociating into channel $n \rightarrow(n-m)+m$ and the total number of dissociative trajectories. This method monitors all fragmentation channels, ensures that the total dissociation rate constant is the sum of the individual channel rate constants and allows comparing various dissociation channels.

\section{Results}

\section{Fragmentation of clusters}

Fragmentation of clusters was analyzed for each cluster size in order to calculate their proper rate constants. Fig. 2 shows the corresponding rate constants for cluster fragmentation of a cluster size 20 in dependence on temperature. The most probable dissociation channel is the emission of single beryllium atoms from the cluster. The formation of dimers $(m=2)$ or trimers $(m=3)$ is not probable. Moreover, the probability of cluster emission decreases with the increasing size of the cluster. This is true for each cluster as shown in Fig. 3.

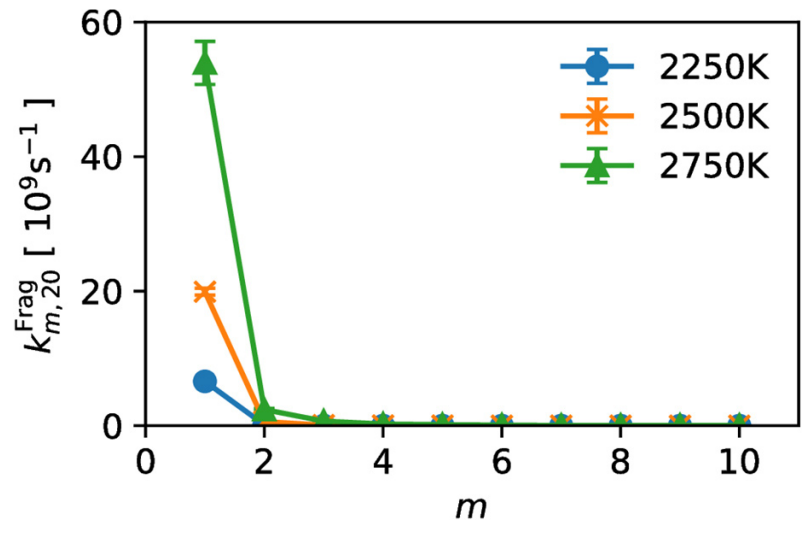

Fig. 2. Fragmentation rate constants, $k_{m, n}^{\text {Frag }}$, of the fragmentation reaction $\mathrm{Be}_{20} \rightarrow \mathrm{Be}_{m}+\mathrm{Be}_{20-m}$ as a function of fragment $m$ for temperatures $2250 \mathrm{~K}$, $2500 \mathrm{~K}$ and $2750 \mathrm{~K}$. The most significant

fragmentation channel is emission of single atoms for every studied temperature. Probability of fragmentation into different channels decreased with the size of the fragment.

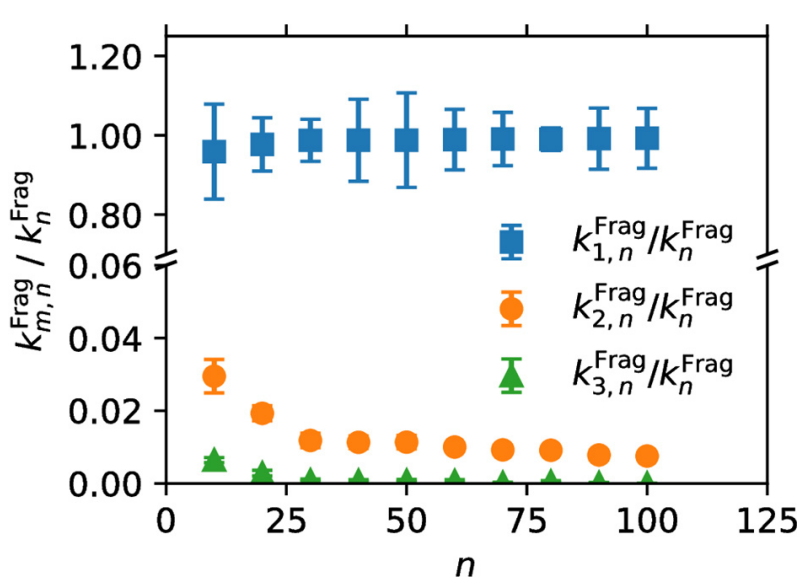

Fig. 3. Ratio of rate constants corresponding to fragmentation of a single atom $\left(k_{1, n}^{\text {Frag }}\right)$, a dimer $\left(k_{2, n}^{\text {Frag }}\right)$ and trimer $\left(k_{3, n}^{\text {Frag }}\right)$ to the total rate constant $\left(k_{n}^{\text {Frag }}\right)$ for Ben clusters, $\mathrm{n} \geq 10$, at $2250 \mathrm{~K}$.

The most significant fragmentation channel is the emission of single atoms for each studied cluster size.

The dependence of rate constants of single atom detachment on temperature is presented in Fig. 4 in the linear form as a function of $\ln \left(k_{1, n}^{\text {Frag }}\right)=\mathrm{f}(1 / \mathrm{T})$ for clusters consisting of 3,10 and 20 Be atoms. This dependence is linear and the Arrhenius equation was used to extrapolate the activation energies for individual processes. Activation energies, $E_{\mathrm{A}}$, obtained for channel $n \rightarrow 1+(n-1)$ for each cluster are given in Fig. 5. All studied clusters have very similar activation energy and stability. Activation energy is almost constant for the cluster size from 5 to 20 atoms and it is increasing slowly from $2.0 \mathrm{eV}$ to $2.5 \mathrm{eV}$ for cluster size greater than 20 atoms. The 
only exception was the fragmentation of the dimer and trimer; their activation energy was significantly lower.

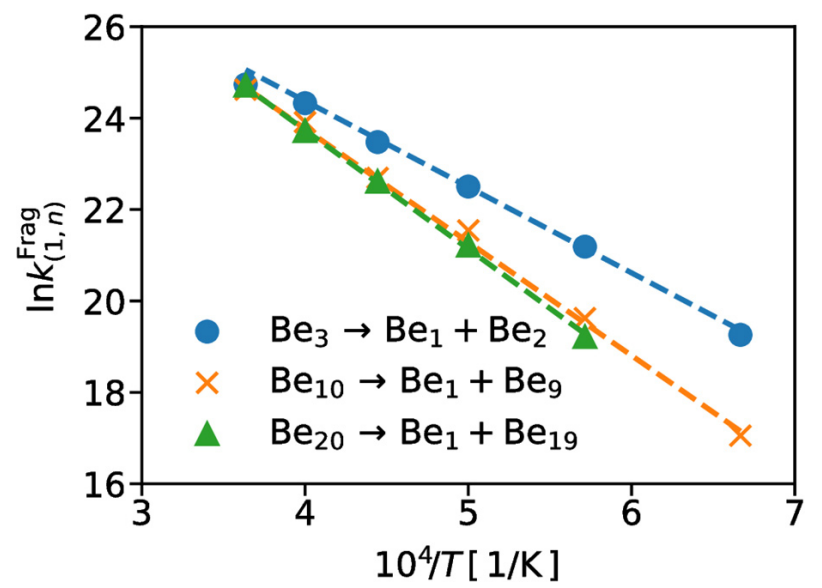

Fig. 4. Dependence of rate constant corresponding to the fragmentation of single atom $\left(k_{1, n}^{\mathrm{Frag}}\right)$ on temperature for clusters $\mathrm{Be}_{3}, \mathrm{Be}_{10}$ and $\mathrm{Be}_{20}$. Dotted line is the linear fit to obtain activation energy.

Total dissociation rate constant depends weakly on the cluster size (see Fig. 6), but a small variation of the total dissociation rate constant can be observed at higher temperatures $(2750 \mathrm{~K})$ and for big clusters. This contradicts the activation energy analysis of the single atom dissociation (reaction channel $n \rightarrow 1+(n-1))$. An increase of the activation energy leads to a lower reaction rate of this channel. Therefore, since the total dissociation rate increases, the reaction rates of other reaction channels become more significant at higher temperatures. Calculated dissociation rate constants for cluster size of 2-20 are given in Tab. $1-5$ at the end of the article. Dissociation rate constants of the cluster size of up to 100 atoms will be provided by the corresponding author upon request.

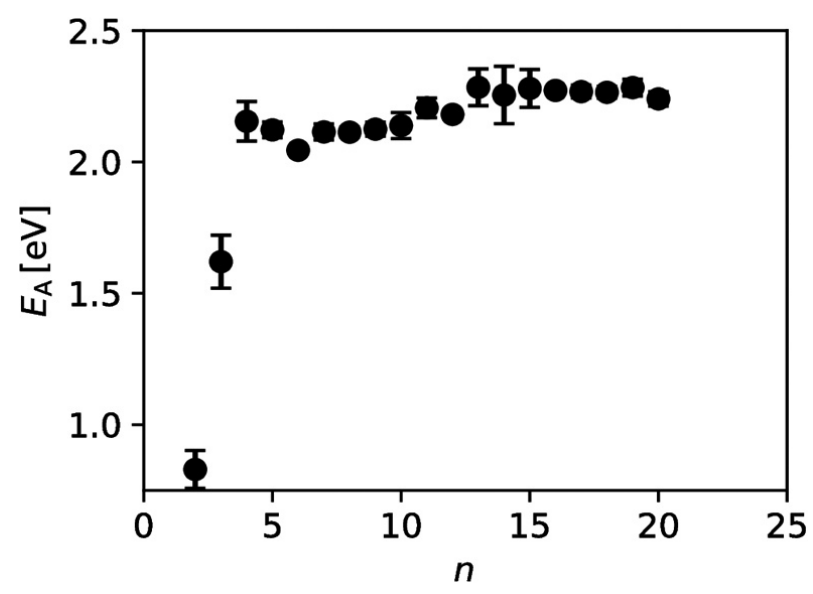

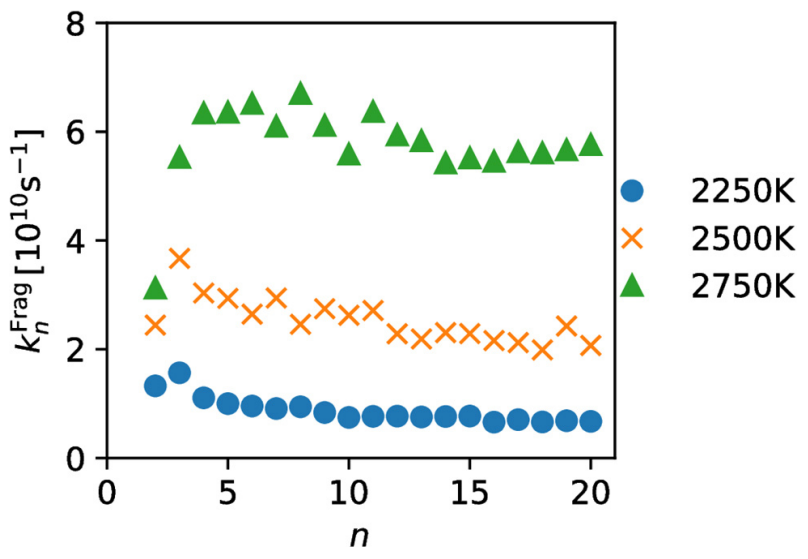

Fig. 6a. Dependence of total dissociation rate constants on the size of the initial cluster at temperatures $2250 \mathrm{~K}, 2500 \mathrm{~K}$ and $2750 \mathrm{~K}$. Cluster size varies from 2 to 20 atoms. Uncertainty of the values is lower than the size of the markers.

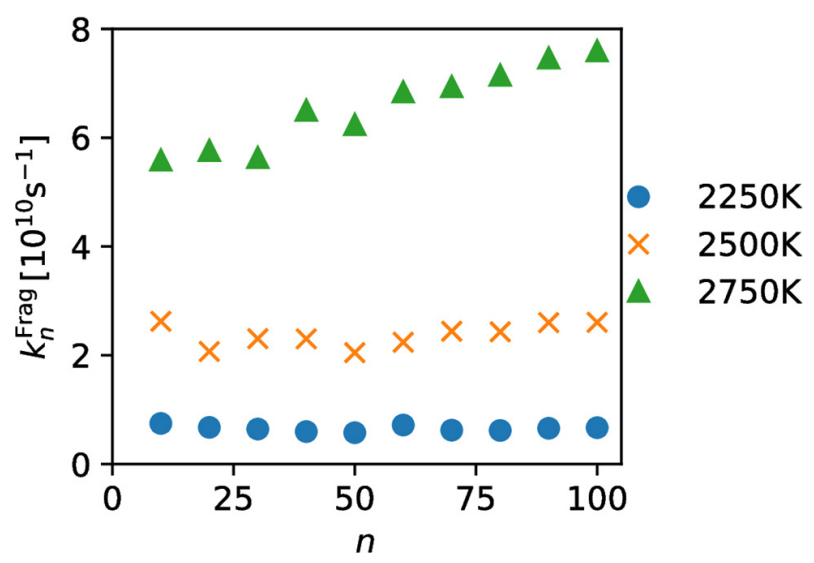

Fig. 6b. Dependence of total dissociation rate constants on the size of the initial cluster at temperatures $2250 \mathrm{~K}, 2500 \mathrm{~K}$ and $2750 \mathrm{~K}$ and for clusters from 10 to 100 atoms. Uncertainty of the values is lower than the size of the markers.

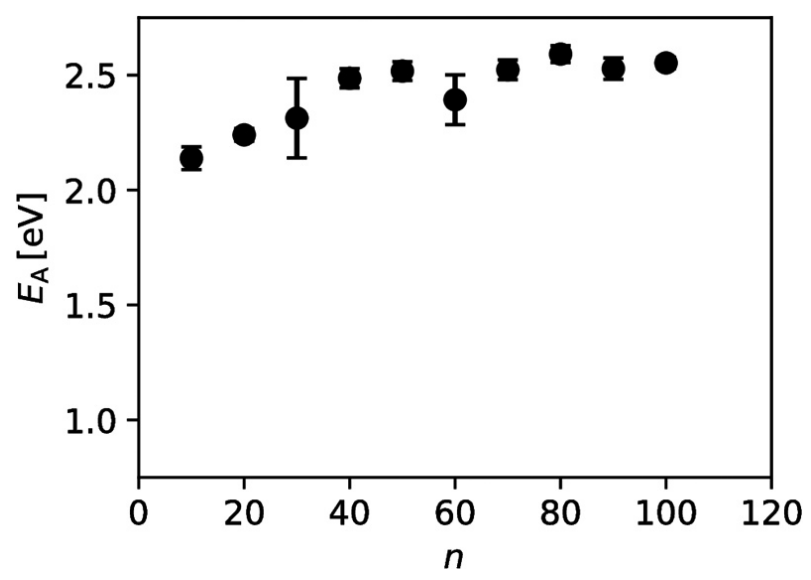

Fig. 5. Activation energy, $E_{\mathrm{A}}$, of single atom detachment obtained from the linear fit of $\ln \left(k_{1, n}^{\text {Frag }}\right)=\mathrm{f}(1 / \mathrm{T})$ in dependence on the cluster size, $n$. 


\section{Association reactions}

Rate constants for the cluster formation reactions exhibit an increasing trend in dependence on the temperature as it illustrated in Fig. 7 for the formation of clusters $\mathrm{Be}_{10}, \mathrm{Be}_{20}, \mathrm{Be}_{50}$ and $\mathrm{Be}_{100}$. Using the collision theory, the dependence is described by the following formula:

$$
\ln \left(k_{1, n}\right)=A+0.5 \ln (T)-\frac{E_{A}}{k_{B} T}
$$

Where $A$ is a fitting parameter dependent on the size of the particle and on the collision frequency, $T$ is the temperature of the system, $k_{\mathrm{B}}$ is the Boltzmann constant and $E_{\mathrm{A}}$ is the activation energy. This formula allows determining the activation energy for the association reaction by the least square fitting procedure. The activation energy as a function of the product size is presented in Fig. 8. The expected theoretical value of the activation energy for these reactions is zero due to no apparent barrier, which agrees well with our results for smaller clusters $(n<15)$. For bigger clusters, calculations predicted the activation energy of around $0.02 \mathrm{eV}$. This is probably the energy needed to dissociate the bond at the impact site to incorporate an impinging atom. Since the average kinetic energy of beryllium atoms is higher, the efficiency of the reaction depends on its cross section and thus on the size of the target cluster. The smallest clusters had negative values, however, they are affected only at higher temperatures due to the inaccuracy of the method and fitting formula.

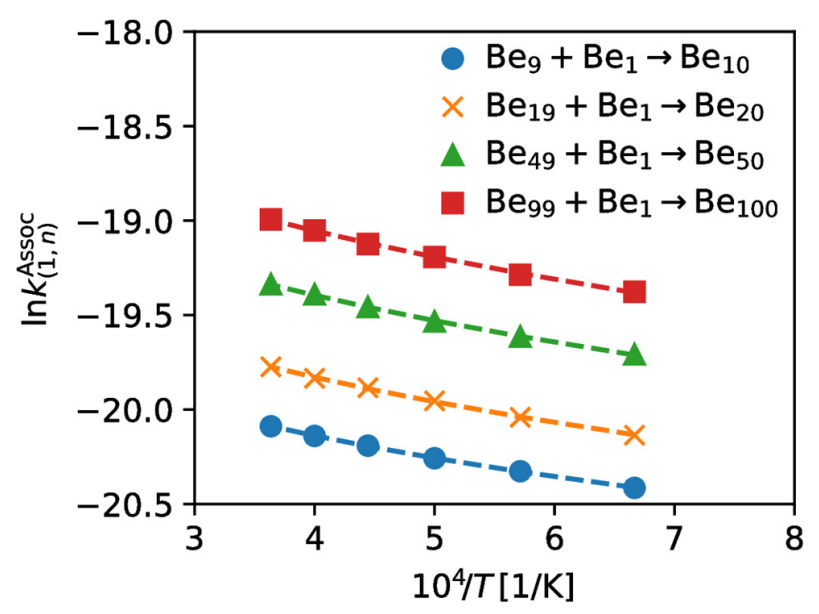

Fig. 7. Dependence of association rate constant, $k_{1, n}^{\text {Assoc }}$, on the temperature for clusters $\mathrm{Be}_{10}, \mathrm{Be}_{20}$, $\mathrm{Be}_{50}$ and $\mathrm{Be}_{100}$. Dashed line is the linear fit defined by Eq. (9) to obtain activation energy of the reaction.

To investigate the influence of the cluster size on the association reaction rate, the dependence of

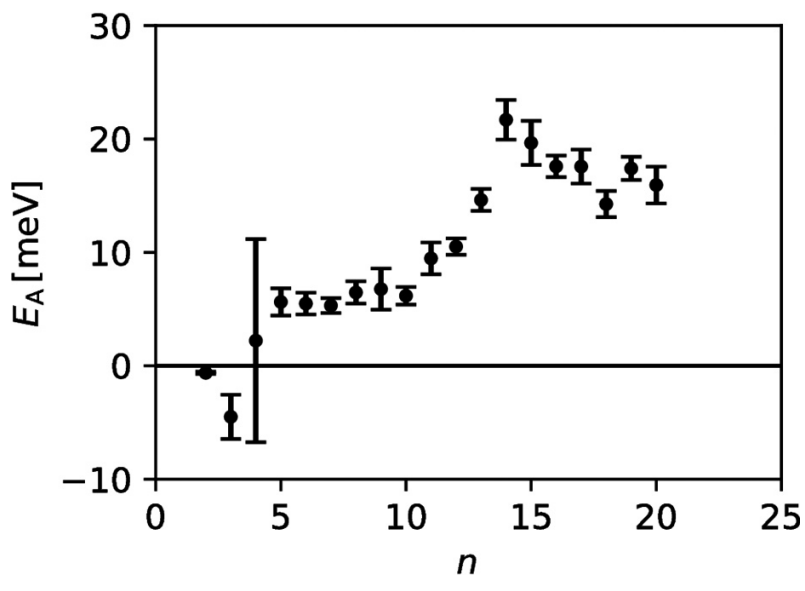

Fig. 8a. Activation energy $E_{\mathrm{A}}$ of the single atom attachment to the cluster as a function of the final cluster size $n$ and for cluster size from 2 to 20 atoms.

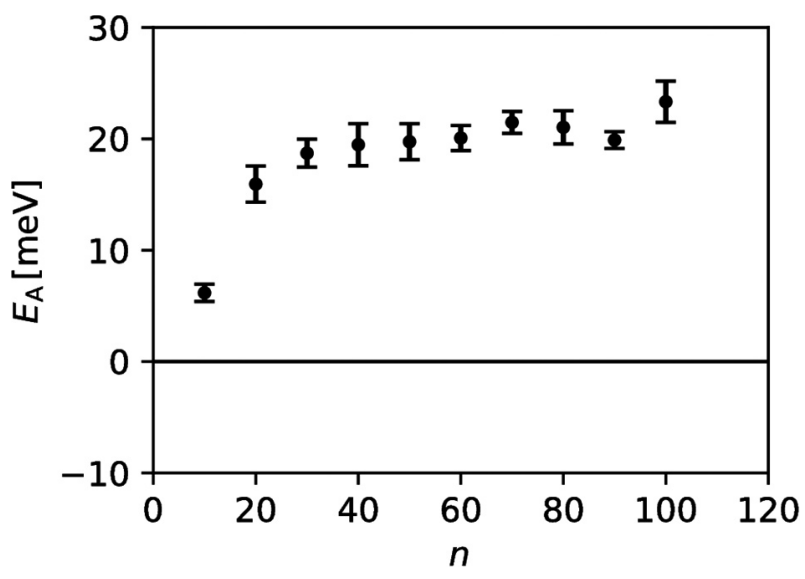

Fig. 8b. Activation energy $E_{\mathrm{A}}$, of the single atom attachment to the cluster as a function of the final cluster size $n$ and for cluster size from 10 to 100 atoms.

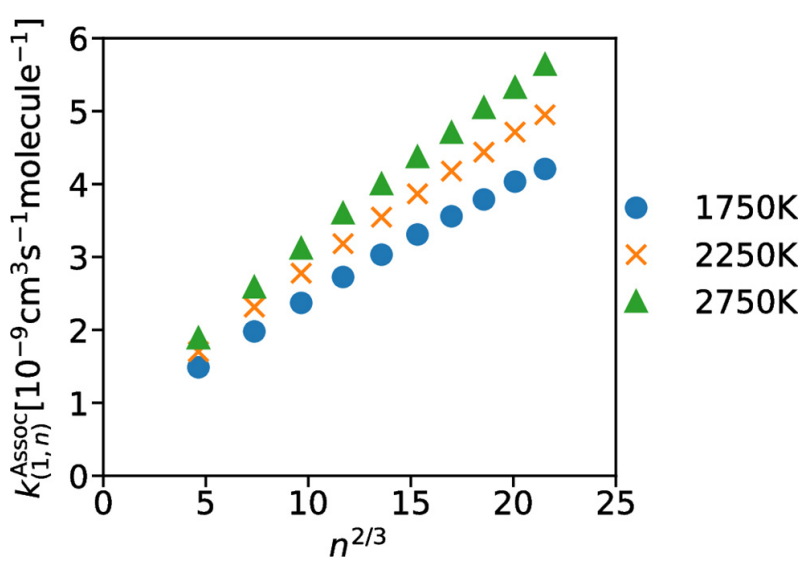

Fig. 9. Association rate constant, $k_{1, n}^{\text {Assoc }}$, as a function of $n^{2 / 3}$ at temperatures of $1750 \mathrm{~K}, 2250 \mathrm{~K}$ and $2750 \mathrm{~K}$. Other temperatures were omitted to preserve clarity. Uncertainty of the values is lower than the size of the marker. 
the rate constant on $n^{2 / 3}$, where $n$ is the number of atoms, was determined and is presented in Fig 9. The reason to plot the rate constant as a function of $n^{2 / 3}$ is to investigate the relation between rate constant and geometric cross section which is proportional to $n^{2 / 3}$ ( $\mathrm{Li}$ and Truhlar, 2008). The observed steep linear dependence proves that the long-range interactions are not included as the used short-range potential was applied. Moreover, the association rate constants for all studied clusters are given in Tab. 6 at the end of the article.

\section{Conclusions}

The present paper reports on the rate constants for fragmentation and association reactions of beryllium clusters consisting of $2-100$ atoms in the temperature range from $1500 \mathrm{~K}$ to $2750 \mathrm{~K}$ determined by the analysis of trajectories obtained by MD simulations with the analytical bond-order interaction potential. Beryllium clusters dissociate mainly into single Be atoms. Their stability depends weakly on cluster size and strongly on temperature. $\mathrm{Be}_{n}$ clusters easily accept single Be atoms while the reaction rate depends mainly on the temperature and size of the cluster.

\section{Acknowledgment}

We are grateful to the HPC center at the Slovak University of Technology in Bratislava, which is a part of the Slovak Infrastructure of High Performance Computing (SIVVP project, ITMS code 26230120002, funded by the European region development funds, ERDF), for the computational time and resources provided.

\section{References}

Björkas C, Juslin N, Timko H, Vörtler K, Nordlund K, Henriksson K, Erhart P (2009) J. Phys. Condens. Matter. 21: 445002.

Blais NC, Truhlar DG, (1983) J. Chem. Phys. 78: 2388-2393.

Brezinsek S (2015) J. Nucl. Mater. 463: 11-21.

Byrd RH, Lu P, Nocedal J, Zhu C (1995) SIAM J. Sci. Comput. 16: 1190-1208.

Doerner RP, Baldwin MJ, Buchenauer D, De Temmerman G, Nishijima D (2009) J. Nucl. Mater. 390-391: 681-684.

Gyoeroek M et al. (2016) Journal of Nuclear Materials. 47: 76-81.

Li ZH, Truhlar DG (2008) J. Phys. Chem. C. 112: 11109-11121.

Litaudon X et al. (2017) Nucl. Fusion. 57: 102001.

Martyna GJ,Klein ML, Tuckerman M, (1992) J. Chem. Phys. 97: 2635-2643.

Matthews GF et al. (2011) Phys. Scr. T145: 014001.

Nishijima D, Doerner RP, Baldwin MJ, De Temmerman G (2009) J. Nucl. Mater. 390-391: 132-135.

Pitts RA, Carpentier S, Escourbiac F, Hirai T, Komarov V, Kukushkin AS, Lisgo S, Loarte A, Merola M, Mitteau R, Raffray AR, Shimada M, Stangeby PC (2011) J. Nucl. Mater. 415: S957-S964.

Safi E et al. (2017) J. Phys. D: Appl. Phys. 50: 204003.

Stillinger FH (1963) J. Chem. Phys. 38: 1486-1494.

Sun Y, Fournier R (2005) Computing Letters. 1: 210-219.

Tuckerman M, Berne BJ, Martyna GJ (1992) J. Chem. Phys. 97: 1990-2001.

Widdowson A, Alves E, Ayres CF, Baron-Wiechec A, Brezinsek S, Catarino N, Coad JP, Heinola K, Likonen J, Matthews GF, Mayer M, Rubel M and JET-EFDA Contributors (2014) Phys Scripta. T159: 014010.

Zheng J, Li Z-H, Jasper AW, Bonhommeau DA, Valero R, Meana-Pañeda R, Mielke SL, Truhlar, DG, ANT, version 2016, University of Minnesota, Minneapolis, 2016. http://comp.chem.umn.edu/ant.

Tab. 1. Dissociation rate constant $k_{m, n}^{\text {Frag }}$ of the reaction $B e_{n} \rightarrow B e_{m}+B e_{n-m}$ at the temperature of $1750 \mathrm{~K}$. All values are in $10^{9} \mathrm{~s}^{-1}$.

\begin{tabular}{|c|c|c|c|c|c|c|c|c|c|c|}
\hline \multirow[b]{2}{*}{$\mathbf{n}$} & \multicolumn{10}{|c|}{$\mathbf{m}$} \\
\hline & 1 & 2 & 3 & 4 & 5 & 6 & 7 & 8 & 9 & 10 \\
\hline 2 & 3.8100 & & & & & & & & & \\
\hline 3 & 1.5905 & & & & & & & & & \\
\hline 4 & 0.5051 & 0.0136 & & & & & & & & \\
\hline 5 & 0.3904 & 0.0065 & & & & & & & & \\
\hline 6 & 0.4395 & 0.0063 & 0.0009 & & & & & & & \\
\hline 7 & 0.3508 & 0.0038 & 0.0002 & & & & & & & \\
\hline 8 & 0.3622 & 0.0038 & 0.0000 & 0.0003 & & & & & & \\
\hline 9 & 0.3477 & 0.0030 & 0.0002 & 0.0002 & & & & & & \\
\hline 10 & 0.3332 & 0.0031 & 0.0003 & 0.0005 & 0.0002 & & & & & \\
\hline 11 & 0.2925 & 0.0015 & 0.0002 & 0.0001 & 0.0001 & & & & & \\
\hline 12 & 0.2680 & 0.0020 & 0.0004 & 0.0001 & 0.0001 & 0.0000 & & & & \\
\hline 13 & 0.2074 & 0.0009 & 0.0002 & 0.0000 & 0.0000 & 0.0000 & & & & \\
\hline 14 & 0.2245 & 0.0006 & 0.0000 & 0.0000 & 0.0000 & 0.0001 & 0.0000 & & & \\
\hline 15 & 0.2208 & 0.0007 & 0.0001 & 0.0000 & 0.0000 & 0.0000 & 0.0000 & & & \\
\hline 16 & 0.2220 & 0.0007 & 0.0001 & 0.0000 & 0.0000 & 0.0000 & 0.0000 & 0.0000 & & \\
\hline 17 & 0.2178 & 0.0018 & 0.0000 & 0.0000 & 0.0001 & 0.0000 & 0.0000 & 0.0000 & & \\
\hline 18 & 0.2146 & 0.0009 & 0.0000 & 0.0000 & 0.0001 & 0.0000 & 0.0000 & 0.0000 & 0.0000 & \\
\hline 19 & 0.2146 & 0.0002 & 0.0000 & 0.0000 & 0.0000 & 0.0000 & 0.0000 & 0.0000 & 0.0000 & \\
\hline 20 & 0.2207 & 0.0007 & 0.0000 & 0.0000 & 0.0000 & 0.0000 & 0.0000 & 0.0000 & 0.0000 & 0.0000 \\
\hline
\end{tabular}


Tab. 2. Dissociation rate constant $k_{m, n}^{\text {Frag }}$ of the reaction $B e_{n} \rightarrow B e_{m}+B e_{n-m}$ at the temperature of $2000 \mathrm{~K}$. All values are in $10^{9} \mathrm{~s}^{-1}$.

\begin{tabular}{|c|c|c|c|c|c|c|c|c|c|c|}
\hline \multirow[b]{2}{*}{$\mathbf{n}$} & \multicolumn{10}{|c|}{$\mathbf{m}$} \\
\hline & 1 & 2 & 3 & 4 & 5 & 6 & 7 & 8 & 9 & 10 \\
\hline 2 & 6.6082 & & & & & & & & & \\
\hline 3 & 5.9247 & & & & & & & & & \\
\hline 4 & 2.7312 & 0.1493 & & & & & & & & \\
\hline 5 & 2.4915 & 0.0822 & & & & & & & & \\
\hline 6 & 2.3957 & 0.0678 & 0.0118 & & & & & & & \\
\hline 7 & 2.3908 & 0.0644 & 0.0191 & & & & & & & \\
\hline 8 & 2.2389 & 0.0483 & 0.0108 & 0.0021 & & & & & & \\
\hline 9 & 2.1491 & 0.0387 & 0.0049 & 0.0060 & & & & & & \\
\hline 10 & 2.2708 & 0.0520 & 0.0081 & 0.0073 & 0.0017 & & & & & \\
\hline 11 & 1.9173 & 0.0278 & 0.0041 & 0.0020 & 0.0005 & & & & & \\
\hline 12 & 1.6685 & 0.0254 & 0.0035 & 0.0006 & 0.0005 & 0.0003 & & & & \\
\hline 13 & 1.7627 & 0.0220 & 0.0033 & 0.0022 & 0.0000 & 0.0007 & & & & \\
\hline 14 & 1.7372 & 0.0169 & 0.0022 & 0.0001 & 0.0006 & 0.0000 & 0.0000 & & & \\
\hline 15 & 1.6851 & 0.0221 & 0.0045 & 0.0012 & 0.0000 & 0.0004 & 0.0000 & & & \\
\hline 16 & 1.3972 & 0.0131 & 0.0022 & 0.0010 & 0.0000 & 0.0000 & 0.0000 & 0.0000 & & \\
\hline 17 & 1.4542 & 0.0134 & 0.0031 & 0.0010 & 0.0005 & 0.0000 & 0.0000 & 0.0000 & & \\
\hline 18 & 1.3939 & 0.0086 & 0.0019 & 0.0003 & 0.0000 & 0.0004 & 0.0000 & 0.0000 & 0.0000 & \\
\hline 19 & 1.4531 & 0.0118 & 0.0013 & 0.0008 & 0.0000 & 0.0000 & 0.0000 & 0.0000 & 0.0000 & \\
\hline 20 & 1.6282 & 0.0151 & 0.0009 & 0.0017 & 0.0000 & 0.0000 & 0.0000 & 0.0000 & 0.0000 & 0.0000 \\
\hline
\end{tabular}

Tab. 3. Dissociation rate constant $k_{m, n}^{\text {Frag }}$ of the reaction $B e_{n} \rightarrow B e_{m}+B e_{n-m}$ at the temperature of $2250 \mathrm{~K}$. All values are in $10^{9} \mathrm{~s}^{-1}$.

\begin{tabular}{|c|c|c|c|c|c|c|c|c|c|c|}
\hline \multirow[b]{2}{*}{$\mathbf{n}$} & \multicolumn{10}{|c|}{$\mathbf{m}$} \\
\hline & 1 & 2 & 3 & 4 & 5 & 6 & 7 & 8 & 9 & 10 \\
\hline 2 & 13.2729 & & & & & & & & & \\
\hline 3 & 15.6802 & & & & & & & & & \\
\hline 4 & 10.3938 & 0.6853 & & & & & & & & \\
\hline 5 & 9.3964 & 0.6160 & & & & & & & & \\
\hline 6 & 9.0484 & 0.4520 & 0.0715 & & & & & & & \\
\hline 7 & 8.5615 & 0.4135 & 0.1297 & & & & & & & \\
\hline 8 & 8.8913 & 0.3730 & 0.1265 & 0.0437 & & & & & & \\
\hline 9 & 7.9812 & 0.2739 & 0.0763 & 0.0440 & & & & & & \\
\hline 10 & 7.1628 & 0.2203 & 0.0480 & 0.0261 & 0.0138 & & & & & \\
\hline 11 & 7.3514 & 0.2284 & 0.0475 & 0.0136 & 0.0256 & & & & & \\
\hline 12 & 7.3657 & 0.2563 & 0.0438 & 0.0183 & 0.0167 & 0.0092 & & & & \\
\hline 13 & 7.2927 & 0.1790 & 0.0409 & 0.0183 & 0.0093 & 0.0135 & & & & \\
\hline 14 & 7.3981 & 0.1707 & 0.0328 & 0.0127 & 0.0136 & 0.0085 & 0.0055 & & & \\
\hline 15 & 7.4933 & 0.1805 & 0.0259 & 0.0125 & 0.0031 & 0.0060 & 0.0021 & & & \\
\hline 16 & 6.4104 & 0.1634 & 0.0204 & 0.0069 & 0.0034 & 0.0041 & 0.0000 & 0.0018 & & \\
\hline 17 & 6.8722 & 0.1594 & 0.0168 & 0.0082 & 0.0049 & 0.0019 & 0.0007 & 0.0030 & & \\
\hline 18 & 6.4857 & 0.1335 & 0.0105 & 0.0074 & 0.0007 & 0.0006 & 0.0005 & 0.0007 & 0.0000 & \\
\hline 19 & 6.7290 & 0.1065 & 0.0200 & 0.0048 & 0.0039 & 0.0003 & 0.0000 & 0.0000 & 0.0000 & \\
\hline 20 & 6.5906 & 0.1301 & 0.0192 & 0.0033 & 0.0028 & 0.0000 & 0.0000 & 0.0000 & 0.0000 & 0.0000 \\
\hline
\end{tabular}

Tab. 4. Dissociation rate constant $k_{m, n}^{\mathrm{Frag}}$ of the reaction $B e_{n} \rightarrow B e_{m}+B e_{n-m}$ at the temperature of $2500 \mathrm{~K}$. All values are in $10^{9} \mathrm{~s}^{-1}$.

\begin{tabular}{|c|c|c|c|c|c|c|c|c|c|c|}
\hline \multirow[b]{2}{*}{$\mathbf{n}$} & \multicolumn{10}{|c|}{$\mathbf{m}$} \\
\hline & 1 & 2 & 3 & 4 & 5 & 6 & 7 & 8 & 9 & 10 \\
\hline 2 & 24.4454 & & & & & & & & & \\
\hline 3 & 36.7097 & & & & & & & & & \\
\hline 4 & 27.6314 & 2.7181 & & & & & & & & \\
\hline 5 & 26.7619 & 2.6073 & & & & & & & & \\
\hline 6 & 24.2031 & 1.8112 & 0.4416 & & & & & & & \\
\hline 7 & 26.7743 & 2.0153 & 0.6441 & & & & & & & \\
\hline 8 & 22.6247 & 1.4182 & 0.3921 & 0.1654 & & & & & & \\
\hline 9 & 25.4156 & 1.3140 & 0.4714 & 0.2613 & & & & & & \\
\hline 10 & 24.2720 & 1.2624 & 0.3638 & 0.2480 & 0.1035 & & & & & \\
\hline 11 & 25.0204 & 1.3481 & 0.3754 & 0.2123 & 0.1798 & & & & & \\
\hline 12 & 21.4032 & 0.9499 & 0.2458 & 0.1232 & 0.0992 & 0.0349 & & & & \\
\hline 13 & 20.5411 & 0.9045 & 0.2085 & 0.1112 & 0.0802 & 0.0472 & & & & \\
\hline 14 & 21.7296 & 0.9306 & 0.1991 & 0.0746 & 0.0803 & 0.0270 & 0.0257 & & & \\
\hline 15 & 21.6192 & 0.8787 & 0.2046 & 0.0691 & 0.0508 & 0.0476 & 0.0183 & & & \\
\hline 16 & 20.6080 & 0.6953 & 0.1325 & 0.0567 & 0.0330 & 0.0376 & 0.0287 & 0.0138 & & \\
\hline 17 & 20.2977 & 0.6483 & 0.1460 & 0.0619 & 0.0386 & 0.0142 & 0.0106 & 0.0142 & & \\
\hline 18 & 19.0443 & 0.6234 & 0.1119 & 0.0530 & 0.0323 & 0.0078 & 0.0150 & 0.0091 & 0.0000 & \\
\hline 19 & 23.2016 & 0.8158 & 0.1539 & 0.0431 & 0.0143 & 0.0118 & 0.0123 & 0.0162 & 0.0049 & \\
\hline 20 & 19.9205 & 0.6060 & 0.0871 & 0.0372 & 0.0228 & 0.0040 & 0.0080 & 0.0098 & 0.0041 & 0.0023 \\
\hline
\end{tabular}


Tab. 5. Dissociation rate constant $k_{m, n}^{\text {Frag }}$ of the reaction $B e_{n} \rightarrow B e_{m}+B e_{n-m}$ at the temperature of $2750 \mathrm{~K}$. All values are in $10^{9} \mathrm{~s}^{-1}$.

\begin{tabular}{|c|c|c|c|c|c|c|c|c|c|c|}
\hline \multirow[b]{2}{*}{$\mathbf{n}$} & \multicolumn{10}{|c|}{$\mathbf{m}$} \\
\hline & 1 & 2 & 3 & 4 & 5 & 6 & 7 & 8 & 9 & 10 \\
\hline 2 & 31.2634 & & & & & & & & & \\
\hline 3 & 55.2716 & & & & & & & & & \\
\hline 4 & 56.8084 & 6.6330 & & & & & & & & \\
\hline 5 & 56.3329 & 7.2518 & & & & & & & & \\
\hline 6 & 57.1485 & 6.4394 & 1.6039 & & & & & & & \\
\hline 7 & 53.4680 & 5.1981 & 2.3396 & & & & & & & \\
\hline 8 & 58.3625 & 5.4294 & 2.3276 & 0.9164 & & & & & & \\
\hline 9 & 53.9445 & 4.5015 & 1.6525 & 1.0502 & & & & & & \\
\hline 10 & 49.4376 & 3.9185 & 1.2217 & 0.8531 & 0.4418 & & & & & \\
\hline 11 & 56.7240 & 4.2097 & 1.2485 & 0.8217 & 0.6978 & & & & & \\
\hline 12 & 53.1727 & 3.7167 & 1.1413 & 0.6481 & 0.4896 & 0.2325 & & & & \\
\hline 13 & 52.8513 & 3.3385 & 1.0289 & 0.5035 & 0.2905 & 0.3164 & & & & \\
\hline 14 & 48.7329 & 3.5225 & 0.8566 & 0.4426 & 0.2937 & 0.3137 & 0.0937 & & & \\
\hline 15 & 50.1926 & 3.2197 & 0.7811 & 0.3912 & 0.2900 & 0.1701 & 0.1301 & & & \\
\hline 16 & 50.2702 & 2.7954 & 0.6938 & 0.3793 & 0.1827 & 0.1023 & 0.0876 & 0.0416 & & \\
\hline 17 & 51.8038 & 2.9573 & 0.6554 & 0.3245 & 0.1667 & 0.1297 & 0.1510 & 0.1064 & & \\
\hline 18 & 52.1026 & 2.6669 & 0.6088 & 0.2848 & 0.1236 & 0.1088 & 0.1001 & 0.0672 & 0.0265 & \\
\hline 19 & 52.5153 & 2.8608 & 0.7315 & 0.2203 & 0.1297 & 0.0638 & 0.0332 & 0.0391 & 0.0795 & \\
\hline 20 & 53.9370 & 2.4003 & 0.6793 & 0.2505 & 0.1509 & 0.0854 & 0.0248 & 0.0434 & 0.0413 & 0.0214 \\
\hline
\end{tabular}

Tab. 6. Association rate constant $k_{1, n}^{\text {Assoc }}$ of the reaction $B e_{1}+B e_{m-1} \rightarrow B e_{n}$ at the temperature of $1500 \mathrm{~K}, 1750 \mathrm{~K}$ $2000 \mathrm{~K} 2250 \mathrm{~K}$ and $2500 \mathrm{~K}$. All values are in $10^{-9} \mathrm{~cm}^{3} \mathrm{~s}^{-1}$ per molecule.

\begin{tabular}{|c|c|c|c|c|c|c|}
\hline \multirow[b]{2}{*}{$\mathbf{n}$} & \multicolumn{6}{|c|}{$\mathbf{T}[\mathbf{K}]$} \\
\hline & 1500 & 1750 & 2000 & 2250 & 2500 & 2750 \\
\hline 2 & 0.7033 & 0.7594 & 0.8116 & 0.8604 & 0.9064 & 0.9501 \\
\hline 3 & 0.8769 & 0.9491 & 1.0089 & 1.0763 & 1.1290 & 1.1696 \\
\hline 4 & 0.9644 & 1.0433 & 1.1164 & 1.1894 & 1.2550 & 1.2780 \\
\hline 5 & 1.0277 & 1.1114 & 1.2009 & 1.2781 & 1.3492 & 1.4172 \\
\hline 6 & 1.1035 & 1.1987 & 1.2885 & 1.3717 & 1.4526 & 1.5166 \\
\hline 7 & 1.1768 & 1.2766 & 1.3658 & 1.4583 & 1.5455 & 1.6199 \\
\hline 8 & 1.2378 & 1.3469 & 1.4467 & 1.5484 & 1.6280 & 1.7189 \\
\hline 9 & 1.3048 & 1.4089 & 1.5251 & 1.6243 & 1.7147 & 1.8129 \\
\hline 10 & 1.3618 & 1.4873 & 1.5934 & 1.7029 & 1.7931 & 1.8871 \\
\hline 11 & 1.4214 & 1.5420 & 1.6645 & 1.7849 & 1.8775 & 1.9915 \\
\hline 12 & 1.4726 & 1.6012 & 1.7341 & 1.8471 & 1.9608 & 2.0595 \\
\hline 13 & 1.4982 & 1.6499 & 1.7757 & 1.9149 & 2.0222 & 2.1336 \\
\hline 14 & 1.5089 & 1.6901 & 1.8414 & 1.9699 & 2.1073 & 2.2149 \\
\hline 15 & 1.5782 & 1.7416 & 1.8882 & 2.0382 & 2.1687 & 2.2728 \\
\hline 16 & 1.6205 & 1.7933 & 1.9455 & 2.0937 & 2.2166 & 2.3361 \\
\hline 17 & 1.6824 & 1.8396 & 1.9993 & 2.1436 & 2.2968 & 2.4134 \\
\hline 18 & 1.7334 & 1.8929 & 2.0469 & 2.2007 & 2.3408 & 2.4624 \\
\hline 19 & 1.7695 & 1.9376 & 2.0958 & 2.2660 & 2.3966 & 2.5416 \\
\hline 20 & 1.8002 & 1.9791 & 2.1585 & 2.3138 & 2.4329 & 2.5849 \\
\hline 30 & 2.1655 & 2.3707 & 2.5838 & 2.7777 & 2.9632 & 3.1191 \\
\hline 40 & 2.4783 & 2.7257 & 2.9722 & 3.1826 & 3.4085 & 3.5993 \\
\hline 50 & 2.7597 & 3.0319 & 3.2859 & 3.5463 & 3.7925 & 3.9996 \\
\hline 60 & 3.0047 & 3.3086 & 3.5947 & 3.8673 & 4.1366 & 4.3711 \\
\hline 70 & 3.2353 & 3.5584 & 3.8680 & 4.1778 & 4.4496 & 4.7048 \\
\hline 80 & 3.4575 & 3.7894 & 4.1328 & 4.4380 & 4.7437 & 5.0466 \\
\hline 90 & 3.6719 & 4.0340 & 4.3897 & 4.7128 & 5.0210 & 5.3229 \\
\hline 100 & 3.8461 & 4.2077 & 4.6205 & 4.9503 & 5.3182 & 5.6377 \\
\hline
\end{tabular}

